\section{Colonic perforation following endoscopic mucosal resection in a patient on bevacizumab treatment}

An 81-year-old woman who had previously undergone left hemicolectomy for an adenocarcinoma (pT3 N1) of the descending colon was diagnosed with a $2-\mathrm{cm}$ flat polyp in the transverse colon during surveillance colonoscopy ( $\bullet$ Fig. 1 a). Subsequently, she also developed liver metastases and underwent three cycles of neoadjuvant chemotherapy with leucovorin, 5-fluorouracil, irinotecan, and bevacizumab (325 mg per cycle).

Five days following the last infusion, she underwent colonoscopy with piecemeal endoscopic mucosal resection (EMR) of the polyp. The polyp margins were treated with hot biopsy forceps ( $\bullet$ Fig. $\mathbf{~ b}$, - Fig. 1c). A few hours post-EMR, the patient developed abdominal pain and signs of localized peritonitis. An urgent computed tomography (CT) scan showed severe edema around the EMR site and air in the mesocolon, but no free air ( $\bullet$ Video 1 ). The patient underwent emergency laparotomy, which revealed a $10-\mathrm{cm}$ necrotic segment in the transverse colon distal to the EMR site. A perforation site was noted at the distal end of the necrotic segment but not at the EMR site. Resection of this segment was performed and a stoma was sited. Histopathology of the surgical specimen showed florid ischemic changes ( $\bullet$ Fig. 2). The patient had an uneventful postoperative recovery.

Bevacizumab is a recombinant monoclonal antibody that blocks angiogenesis, thereby inhibiting tumor growth. In patients undergoing bevacizumab treatment, perforation risks of $0.9 \%$ in general [1] and $12.5 \%$ following colonic stenting [2] have been reported.

Recent colonoscopy ( $<1$ month) is a risk factor for bevacizumab-related perforation [1]. This case illustrates that bowel ischemia is probably the culprit behind perforation in these patients, as has been previously proposed in patients receiving bevacizumab following radiotherapy [3]. It is conceivable that bevacizumab-related ischemia may have been aggravated by bowel preparation, luminal instrumentation, and adrenalin injection, therefore resulting in ischemia-related bowel perforation. Stopping bevacizumab for at least 28 days before and after surgical procedures is recommended [4], and this would also be reasonable for colonoscopy procedures, unless they are clinically essential.

\section{Endoscopy_UCTN_Code_CPL_1AJ_2AD}

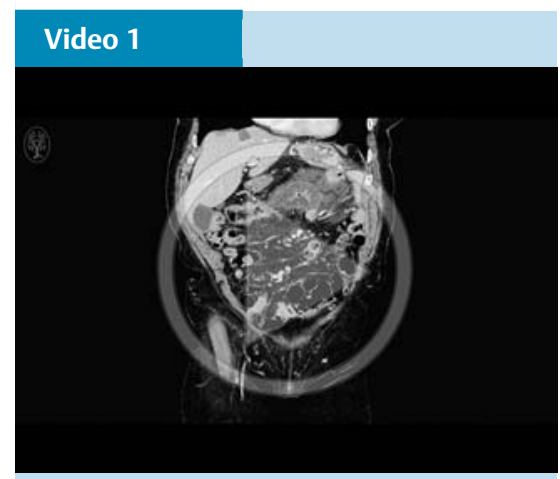

Computed tomography (CT) scan performed a few hours post-endoscopic mucosal resection (EMR) showing severe edema in the colonic wall proximal and distal to the EMR site (identified by the endoscopic clips) and air in the mesocolon distal to the EMR site.
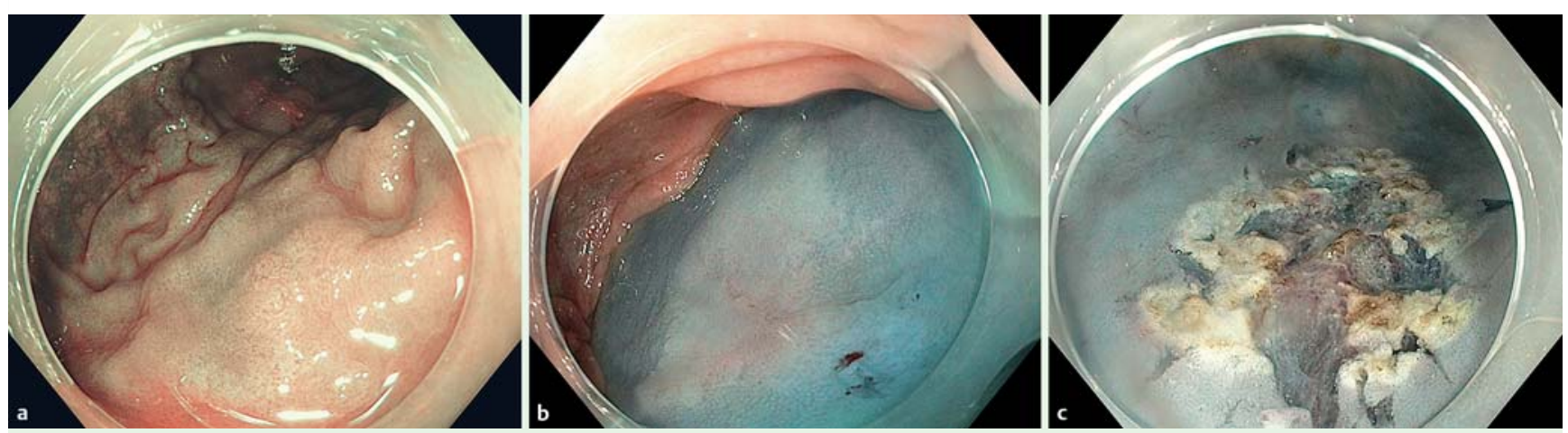

Fig. 1 Endoscopic views of the polyp before and after endoscopic mucosal resection showing: a a flat polyp on narrow-band imaging (Paris classification 0 -Ilb); $\mathbf{b}$ the polyp after injection of $30 \mathrm{ml}$ of a mixture of $0.9 \%$ saline, indigo carmine, and adrenaline (1:10 000); $\mathbf{c}$ the residual ulcer after piecemeal endoscopic resection and treatment of the remnant polypoid tissue at the margins with hot biopsy forceps (performed to minimize the risk of polyp recurrence).
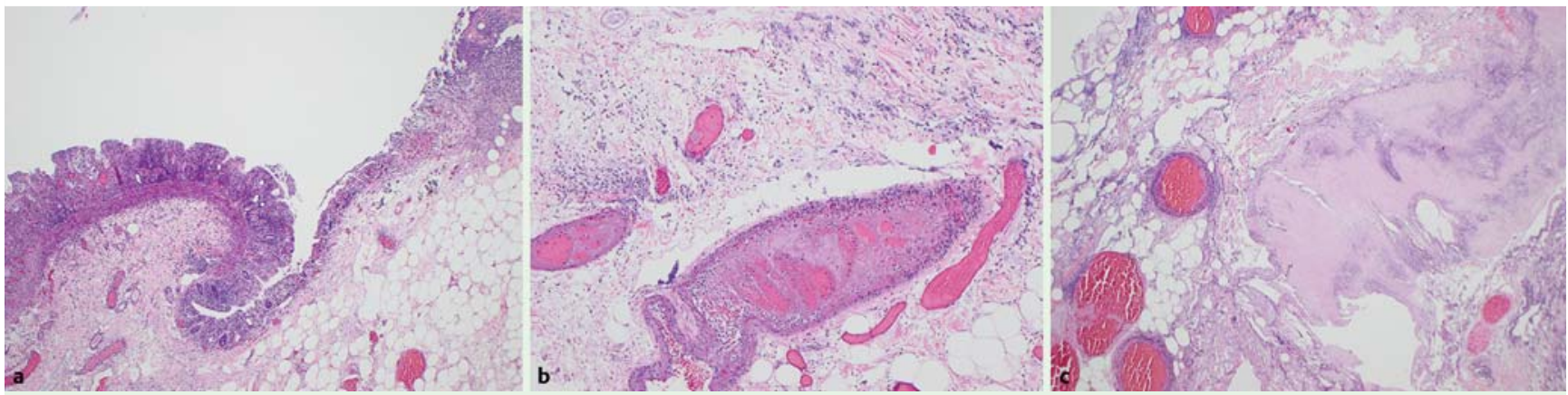

Fig. 2 Histology of the hematoxylin and eosin (H\&E)-stained surgical specimen showed: $\mathbf{a}$ ischemic mucosa; $\mathbf{b}$ thrombosed blood vessels; $\mathbf{c}$ necrosis of the muscular layer. 
Competing interests: Søren Meisner is a consultant for Olympus Europe. Evangelos Kalaitzakis received travel grant form Olympus Europe. The other authors have no conflict of interest to disclose.

\section{Evangelos Kalaitzakis ${ }^{1}$, Roald Flesland Havre $^{1}$, Gro Linno Willemoe ${ }^{2}$, Søren Meisner ${ }^{1}$}

${ }^{1}$ Endoscopy Unit, Copenhagen University Hospital/Herlev, University of Copenhagen, Copenhagen, Denmark

${ }^{2}$ Department of Pathology, Copenhagen University Hospital/Righospitalet, University of Copenhagen, Copenhagen, Denmark

\section{References}

1 Hapani S, Chu D, Wu S. Risk of gastrointestinal perforation in patients with cancer treated with bevacizumab: a meta-analysis. Lancet Oncol 2009; 10: 559-568

2 Van Halsema EE, van Hooft JE, Small AJ. Perforation in colorectal stenting: a meta-analysis and a search for risk factors. Gastrointest Endosc 2014; 79: 970-982

3 Lordick F, Geinitz H, Theisen J et al. Increased risk of ischemic bowel complications during treatment with bevacizumab after pelvic irradiation: report of three cases. Int J Radiation Oncology Biol Phys 2006; 64: 1295 1298

4 Scappaticci FA, Fehrenbacher L, Cartwright T et al. Surgical wound healing complications in metastatic colorectal cancer patients treated with bevacizumab. J Surg Oncol 2005; 91: 173-180
Bibliography

DoI http://dx.doi.org/

10.1055/s-0042-109604

Endoscopy 2016; 48: E224-E225

(c) Georg Thieme Verlag KG

Stuttgart · New York

ISSN 0013-726X

\section{Corresponding author} Evangelos Kalaitzakis, MD, PhD, MSc Endoscopy Unit

Digestive Disease Center

Copenhagen University Hospital/Herlev 2400 Copenhagen

Denmark

kalvag@hotmail.com 